\title{
Two-dimensional soliton cellular automaton of deautonomized Toda-type
}

\author{
A. Nagai ${ }^{1,2}$, T. Tokihiro ${ }^{1}$, J. Satsuma $^{1}$, R. Willox $^{1,3}$ and K. Kajiwara ${ }^{4}$ \\ ${ }^{1}$ Graduate School of Mathematical Sciences, University of Tokyo, \\ Komaba 3-8-1, Meguro-ku, Tokyo 153, Japan \\ ${ }^{3}$ Dienst Tena, Vrije Universiteit Brussel, \\ Pleinlaan 2, 1050 Brussels, Belgium \\ ${ }^{4}$ Department of Electrical Engineering, Doshisha University, \\ Tanabe, Kyoto 610-03, Japan \\ (Received )
}

\begin{abstract}
A deautonomized version of the two-dimensional Toda lattice equation is presented. Its ultra-discrete analogue and soliton solutions are also discussed.

PACS: 03.20.+i; 03.40.Kf; 04.60.Nc

Keywords: deautonomized, ultra-discrete, two-dimensional Toda lattice equation.
\end{abstract}

${ }^{2}$ E-mail: nagai@sat.t.u-tokyo.ac.jp

to appear in Phys. Lett. A. 


\section{Introduction}

The importance of discrete soliton equations has been recognized in many fields such as mathematics, physics and engineering, owing to the enormous development of computer science. Quite recently, two new types of discretization have been proposed. One of these is to unequalize lattice intervals [1], which we call "deautonomization" in this paper. This idea comes from Newton's interpolation formula [2] and now many applications in numerical computation such as in the $\rho$-algorithm and in adaptive numerical integration are expected. Another type of discretization is to discretize dependent as well as independent variables, known as "ultra-discretization". One of the most important ultra-discrete soliton systems is the so-called "soliton cellular automaton" or SCA for short [3, 田. In recent papers [5, 6], a general method to obtain the SCA from discrete soliton equations was proposed. This procedure has been applied to other soliton equations [7] as well.

The aim of this paper is to study a deautonomized version of the two-dimensional(2D) Toda lattice equation and its ultra-discrete analogue, which is considered as a deautonomized version of the 2D Todatype SCA [7. In section 2, we review the result by Hirota et al. on the 2D Toda lattice equation [8] and derive its deautonomized version. In section 3, we present an ultra-discrete analogue of the deautonomized 2D Toda lattice equation, soliton solutions for which are also discussed. Concluding remarks are given in section 4.

\section{Deautonomization of the 2D Toda lattice equation}

We start with a discrete analogue of the 2D Toda lattice equation in bilinear form [8,

$$
\begin{aligned}
& \left(\Delta_{l}^{+} \Delta_{m}^{-} \tau(l, m, n)\right) \tau(l, m, n)-\left(\Delta_{l}^{+} \tau(l, m, n)\right) \Delta_{m}^{-} \tau(l, m, n) \\
= & \tau(l, m-1, n+1) \tau(l+1, m, n-1)-\tau(l+1, m-1, n) \tau(l, m, n), l, m, n \in \mathbf{Z},
\end{aligned}
$$

where $\Delta_{l}^{+}$and $\Delta_{m}^{-}$stand for difference operators defined by

$$
\Delta_{l}^{+} \tau=\frac{\tau(l+1, m, n)-\tau(l, m, n)}{\delta}, \Delta_{m}^{-} \tau=\frac{\tau(l, m, n)-\tau(l, m-1, n)}{\epsilon} .
$$

Rewriting eq. (1) by using eq. (2), we obtain

$$
\begin{array}{r}
(1-\delta \epsilon) \tau(l+1, m-1, n) \tau(l, m, n)-\tau(l+1, m, n) \tau(l, m-1, n) \\
+\delta \epsilon \tau(l, m-1, n+1) \tau(l+1, m, n-1)=0,
\end{array}
$$

which possesses a particular solution expressed as [8]

$$
\tau(l, m, n)=\left|\begin{array}{cccc}
f^{(1)}(l, m, n) & f^{(1)}(l, m, n+1) & \cdots & f^{(1)}(l, m, n+N-1) \\
f^{(2)}(l, m, n) & f^{(2)}(l, m, n+1) & \cdots & f^{(2)}(l, m, n+N-1) \\
\vdots & \vdots & & \vdots \\
f^{(N)}(l, m, n) & f^{(N)}(l, m, n+1) & \cdots & f^{(N)}(l, m, n+N-1)
\end{array}\right| .
$$

In eq. (四) each entry $f^{(i)}(l, m, n)(i=1,2, \cdots, N)$ satisfies the following dispersion relations;

$$
\left\{\begin{array}{l}
\Delta_{l}^{+} f^{(i)}(l, m, n)=f^{(i)}(l, m, n+1), \\
\Delta_{m}^{-} f^{(i)}(l, m, n)=-f^{(i)}(l, m, n-1) .
\end{array}\right.
$$


When we take

$$
f^{(i)}(l, m, n)=p_{i}^{n}\left(1+\delta p_{i}\right)^{l}\left(1+\epsilon p_{i}^{-1}\right)^{-m}+q_{i}^{n}\left(1+\delta q_{i}\right)^{l}\left(1+\epsilon q_{i}^{-1}\right)^{-m}
$$

as a solution for eq. (5), the $\tau$-function (4) gives an $N$-soliton solution.

As opposed to the autonomous eq. (1), where there is a constant lattice interval in each direction, we now present a deautonomized version, where lattice interval between neighboring lattice points can be chosen freely. Let us replace $f^{(i)}(l, m, n)$ in eq. (6) by

$$
\begin{array}{r}
f^{(i)}\left(l_{t}, m_{j}, n\right)=p_{i}^{n} \frac{\prod_{a}^{t-1}\left(1+\delta_{a} p_{i}\right) \quad}{\prod_{b}^{j-1}\left(1+\epsilon_{b} p_{i}^{-1}\right)}+q_{i}^{n} \frac{\prod_{a}^{t-1}\left(1+\delta_{a} q_{i}\right)}{\prod_{b}^{j-1}\left(1+\epsilon_{b} q_{i}^{-1}\right)}, \\
\delta_{t}=l_{t+1}-l_{t}, \epsilon_{j}=m_{j+1}-m_{j}, t, j \in \mathbf{Z},
\end{array}
$$

where the products represent, for example,

$$
\prod_{a}^{t-1}\left(1+\delta_{a} p_{i}\right)= \begin{cases}\prod_{a=0}^{t-1}\left(1+\delta_{a} p_{i}\right) & t \geq 1 \\ 1 & t=0 \\ \prod_{a=t}^{-1}\left(1+\delta_{a} p_{i}\right)^{-1} & t \leq-1\end{cases}
$$

Then the following dispersion relations hold;

$$
\left\{\begin{array}{c}
\Delta_{l_{t}}^{+} f^{(i)}\left(l_{t}, m_{j}, n\right) \equiv \frac{f^{(i)}\left(l_{t+1}, m_{j}, n\right)-f^{(i)}\left(l_{t}, m_{j}, n\right)}{\delta_{t}}=f^{(i)}\left(l_{t}, m_{j}, n+1\right), \\
\Delta_{m_{j}}^{-} f^{(i)}\left(l_{t}, m_{j}, n\right) \equiv \frac{f^{(i)}\left(l_{t}, m_{j}, n\right)-f^{(i)}\left(l_{t}, m_{j-1}, n\right)}{\epsilon_{j-1}}=-f^{(i)}\left(l_{t}, m_{j}, n-1\right) .
\end{array}\right.
$$

By using the Plücker identity for determinants, we see that the $\tau$-function given by

$$
\tau\left(l_{t}, m_{j}, n\right)=\left|\begin{array}{cccc}
f^{(1)}\left(l_{t}, m_{j}, n\right) & f^{(1)}\left(l_{t}, m_{j}, n+1\right) & \cdots & f^{(1)}\left(l_{t}, m_{j}, n+N-1\right) \\
f^{(2)}\left(l_{t}, m_{j}, n\right) & f^{(2)}\left(l_{t}, m_{j}, n+1\right) & \cdots & f^{(2)}\left(l_{t}, m_{j}, n+N-1\right) \\
\vdots & \vdots & & \vdots \\
f^{(N)}\left(l_{t}, m_{j}, n\right) & f^{(N)}\left(l_{t}, m_{j}, n+1\right) & \cdots & f^{(N)}\left(l_{t}, m_{j}, n+N-1\right)
\end{array}\right|,
$$

with each $f^{(i)}$ solution of eq. (10), satisfies a bilinear equation,

$$
\begin{aligned}
& \left(\Delta_{l_{t}}^{+} \Delta_{m_{j}}^{-} \tau\left(l_{t}, m_{j}, n\right)\right) \tau\left(l_{t}, m_{j}, n\right)-\Delta_{l_{t}}^{+} \tau\left(l_{t}, m_{j}, n\right) \Delta_{m_{j}}^{-} \tau\left(l_{t}, m_{j}, n\right) \\
= & \tau\left(l_{t}, m_{j-1}, n+1\right) \tau\left(l_{t+1}, m_{j}, n-1\right)-\tau\left(l_{t+1}, m_{j-1}, n\right) \tau\left(l_{t}, m_{j}, n\right),
\end{aligned}
$$

or equivalently,

$$
\begin{array}{r}
\left(1-\delta_{t} \epsilon_{j-1}\right) \tau\left(l_{t+1}, m_{j-1}, n\right) \tau\left(l_{t}, m_{j}, n\right)-\tau\left(l_{t+1}, m_{j}, n\right) \tau\left(l_{t}, m_{j-1}, n\right) \\
+\delta_{t} \epsilon_{j-1} \tau\left(l_{t}, m_{j-1}, n+1\right) \tau\left(l_{t+1}, m_{j}, n-1\right)=0,
\end{array}
$$

which is considered as a deautonomized 2D Toda lattice equation (in the sense explained above). 
It is interesting to note that when we replace $f^{(i)}(l, m, n)$ by

$$
\begin{aligned}
f^{(i)}\left(l_{t}, m_{j}, n_{k}\right) & =\prod_{c}^{k-1}\left(\eta_{c} p_{i}\right) \frac{\prod_{a}^{t-1}\left(1+\delta_{a} p_{i}\right)}{\prod_{b}^{j-1}\left(1+\epsilon_{b} p_{i}^{-1}\right)}+\prod_{c}^{k-1}\left(\eta_{c} q_{i}\right) \frac{\prod_{a}^{t-1}\left(1+\delta_{a} q_{i}\right)}{\prod_{b}^{j-1}\left(1+\epsilon_{b} q_{i}^{-1}\right)}, \\
\eta_{c} & =n_{c+1}-n_{c},
\end{aligned}
$$

we can construct a new bilinear equation deautonomized with respect to all independent variables. However, it reduce to eq. (12) by simple dependent variable transformation.

\section{Ultra-discretization of the deautonomized 2D Toda lattice equation}

In this section, we present an ultra-discrete analogue of the deautonomized Toda lattice eq. (13) and we construct its soliton solutions. Let us denote $\tau\left(l_{t}, m_{j}, n\right)$ as $\tau_{t, j, n}$ for simplicity. Equation (13) is rewritten as

$$
\left(1-\delta_{t} \epsilon_{j}\right) \tau_{t+1, j, n} \tau_{t, j+1, n}-\tau_{t+1, j+1, n} \tau_{t, j, n}+\delta_{t} \epsilon_{j} \tau_{t, j, n+1} \tau_{t+1, j+1, n-1}=0 .
$$

When we introduce the new dependent variable $S_{t, j, n}$ as

$$
\tau_{t, j, n}=\exp \left[S_{t, j, n}\right]
$$

eq. (16) is equivalent to

$$
\begin{array}{r}
\left(1-\delta_{t} \epsilon_{j}\right)+\delta_{t} \epsilon_{j} \exp \left[\mathrm{e}^{-\partial_{n}}\left(\Delta_{n}^{+}-\Delta_{t}^{+}\right)\left(\Delta_{n}^{+}-\Delta_{j}^{+}\right) S_{t, j, n}\right]=\exp \left[\Delta_{t}^{+} \Delta_{j}^{+} S_{t, j, n}\right] \\
\Delta_{t}^{+}=\mathrm{e}^{\partial_{t}}-1, \Delta_{j}^{+}=\mathrm{e}^{\partial_{j}}-1, \Delta_{n}^{+}=\mathrm{e}^{\partial_{n}}-1 .
\end{array}
$$

Let us define a difference operator $\Delta^{\prime}$ by

$$
\Delta^{\prime}=\mathrm{e}^{-\partial_{n}}\left(\Delta_{n}^{+}-\Delta_{t}^{+}\right)\left(\Delta_{n}^{+}-\Delta_{j}^{+}\right)
$$

for convenience sake. Taking a logarithm and operating $\Delta^{\prime}$ on both sides of eq. (18), we have

$$
\Delta^{\prime} \log \left(1-\delta_{t} \epsilon_{j}\right)+\Delta^{\prime} \log \left[1+\frac{\delta_{t} \epsilon_{j}}{1-\delta_{t} \epsilon_{j}} \exp \left(\Delta^{\prime} S_{t, j, n}\right)\right]=\Delta_{t}^{+} \Delta_{j}^{+} \Delta^{\prime} S_{t, j, n}
$$

We finally take an ultra-discrete limit of eq. (21). Setting

$$
\Delta^{\prime} S_{t, j, n}=\frac{v_{t, j, n}^{\varepsilon}}{\varepsilon}, \delta_{t}=\mathrm{e}^{-\theta_{t} / \varepsilon}, \epsilon_{j}=\mathrm{e}^{-\sigma_{j} / \varepsilon}\left(\theta_{t}, \sigma_{j} \in \mathbf{Z}_{\geq 0}\right),
$$

we obtain

$$
\begin{array}{r}
\Delta_{t}^{+} \Delta_{j}^{+} v_{t, j, n}=\mathrm{e}^{-\partial_{n}}\left(\Delta_{n}^{+}-\Delta_{t}^{+}\right)\left(\Delta_{n}^{+}-\Delta_{j}^{+}\right) F\left(v_{t, j, n}-\theta_{t}-\sigma_{j}\right), \\
F(x)=\max (0, x)
\end{array}
$$

in the limit $\varepsilon \rightarrow+0$. We have rewritten $\lim _{\varepsilon \rightarrow+0} v_{t, j, n}^{\varepsilon}$ as $v_{t, j, n}$. Equation (23) is considered as an ultradiscrete analogue of the deautonomized 2D Toda lattice equation. 
Next, we discuss soliton solutions for eq. (23). It is natural to consider that soliton solutions for eq. (23) are obtained by an ultra-discretization of those for eq. (12). First of all, a one-soliton solution for eq. (12) is given by

$$
\begin{aligned}
\tau_{t, j, n} & =1+\eta_{1} \\
\eta_{1} & =\alpha_{1}\left(\frac{p_{1}}{q_{1}}\right)^{n} \prod_{a}^{t-1} \frac{1+\delta_{a} p_{1}}{1+\delta_{a} q_{1}} \prod_{b}^{j-1} \frac{1+\epsilon_{b} q_{1}^{-1}}{1+\epsilon_{b} p_{1}^{-1}} .
\end{aligned}
$$

Introducing new parameters and variable as

$$
\begin{array}{r}
\mathrm{e}^{P_{1} / \varepsilon}=p_{1}, \mathrm{e}^{Q_{1} / \varepsilon}=q_{1}, \mathrm{e}^{A_{1} / \varepsilon}=\alpha_{1}, \\
\mathrm{e}^{-\theta_{t} / \varepsilon}=\delta_{t}, \mathrm{e}^{-\sigma_{j} / \varepsilon}=\epsilon_{j}, \\
\rho_{t, j, n}^{\varepsilon}=\varepsilon \log \tau_{t, j, n}
\end{array}
$$

and taking the limit $\varepsilon \rightarrow+0$, we obtain

$$
\begin{aligned}
\lim _{\varepsilon \rightarrow+0} \rho_{t, j, n}^{\varepsilon} \equiv \rho_{t, j, n}= & \max \left(0, K_{1}\right) \\
K_{1}= & A_{1}+n\left(P_{1}-Q_{1}\right)+\sum_{a}^{t-1}\left\{F\left(P_{1}-\theta_{a}\right)-F\left(Q_{1}-\theta_{a}\right)\right\} \\
& +\sum_{b}^{j-1}\left\{F\left(-Q_{1}-\sigma_{b}\right)-F\left(-P_{1}-\sigma_{b}\right)\right\}, \\
\sum_{a}^{t-1}= & \begin{cases}\sum_{a=0}^{t-1} t \geq 1, \\
0 & t=0, \\
-\sum_{a=t}^{-1} & t \leq-1 .\end{cases}
\end{aligned}
$$

A one-soliton solution for eq. (23) is given by

$$
\begin{aligned}
v_{t, j, n}= & \rho_{t+1, j+1, n-1}+\rho_{t, j, n+1}-\rho_{t+1, j, n}-\rho_{t, j+1, n} \\
= & \max \left(0, K_{1}-P_{1}+Q_{1}+F\left(P_{1}-\theta_{t}\right)-F\left(Q_{1}-\theta_{t}\right)-F\left(-P_{1}-\sigma_{j}\right)+F\left(-Q_{1}-\sigma_{j}\right)\right) \\
& +\max \left(0, K_{1}+P_{1}-Q_{1}\right)-\max \left(0, K_{1}+F\left(P_{1}-\theta_{t}\right)-F\left(Q_{1}-\theta_{t}\right)\right) \\
& -\max \left(0, K_{1}-F\left(-P_{1}-\sigma_{j}\right)+F\left(-Q_{1}-\sigma_{j}\right)\right) .
\end{aligned}
$$

Secondly, we construct a two-soliton solution. Equation (12) admits a two-soliton solution,

$$
\begin{aligned}
\tau_{t, j, n} & =1+\eta_{1}+\eta_{2}+\theta_{12} \eta_{1} \eta_{2} \\
\eta_{i} & =\alpha_{i}\left(\frac{p_{i}}{q_{i}}\right)^{n} \prod_{a}^{t-1} \frac{1+\delta_{a} p_{i}}{1+\delta_{a} q_{i}} \prod_{b}^{j-1} \frac{1+\epsilon_{b} q_{i}^{-1}}{1+\epsilon_{b} p_{i}^{-1}} \quad(i=1,2), \\
\theta_{12} & =\frac{\left(p_{2}-p_{1}\right)\left(q_{1}-q_{2}\right)}{\left(q_{1}-p_{2}\right)\left(q_{2}-p_{1}\right)} .
\end{aligned}
$$

In order to take an ultra-discrete limit for the above solution, we suppose without loss of generality that the inequality,

$$
0<p_{1}<p_{2}<q_{2}<q_{1}
$$


holds. Introducing new parameters and variable as

$$
\begin{aligned}
& \mathrm{e}^{P_{i} / \varepsilon}=p_{i}, \mathrm{e}^{Q_{i} / \varepsilon}=q_{i}, \mathrm{e}^{A_{i} / \varepsilon}=\alpha_{i}\left(i=1,2, P_{1}<P_{2}<Q_{2}<Q_{1}\right), \\
& \rho_{t, j, n}^{\varepsilon}=\varepsilon \log \tau_{t, j, n}
\end{aligned}
$$

and taking the limit of small $\varepsilon$, we obtain

$$
\begin{aligned}
\lim _{\varepsilon \rightarrow+0} \rho_{t, j, n}^{\varepsilon} \equiv \rho_{t, j, n}= & \max \left(0, K_{1}, K_{2}, K_{1}+K_{2}+P_{2}-Q_{2}\right) \\
K_{i}= & A_{i}+n\left(P_{i}-Q_{i}\right)+\sum_{a}^{t-1}\left\{F\left(P_{i}-\theta_{a}\right)-F\left(Q_{i}-\theta_{a}\right)\right\} \\
& +\sum_{b}^{j-1}\left\{F\left(-Q_{i}-\sigma_{b}\right)-F\left(-P_{i}-\sigma_{b}\right)\right\} \quad(i=1,2) .
\end{aligned}
$$

Finally, an $N$-soliton solution can also be constructed, under the assumption that the inequality,

$$
0<p_{1}<p_{2}<\cdots<p_{N}<q_{N}<\cdots<q_{2}<q_{1}
$$

holds. Through the same limiting procedure, we have

$$
\rho_{t, j, n}=\max _{\mu=0,1}\left(\sum_{i=1}^{N} \mu_{i} K_{i}+\sum_{1 \leq i<i^{\prime} \leq N} \mu_{i} \mu_{i^{\prime}}\left(P_{i^{\prime}}-Q_{i^{\prime}}\right)\right),
$$

where $\max _{\mu=0,1}$ is the maximization over all possible combinations of $\mu_{1}=0,1, \mu_{2}=0,1, \cdots, \mu_{N}=0,1$. Since the bounded and uniform convergence of the dependent variable $\rho_{t, j, n}^{\varepsilon}$ as $\varepsilon \rightarrow+0$ is the cornerstone of the ultra-discretization, it should be clear that eq. (44), which is the ultra-discretization of the $N$ soliton solution for the discrete 2D Toda lattice equation, is an $N$-soliton solution for the ultra-discrete equation.

We show one- and two-soliton solutions for eq. (23) with $\sigma_{j}, \theta_{t}$ constant in Figures 1,3 and with $\sigma_{j}, \theta_{t}$ chosen randomly in Figures 2,4. It should be noted that the solutions with $\sigma_{j}, \theta_{t}$ constant are equivalent to those discussed in [7]. We see that arbitrariness of $\sigma_{j}$ and $\theta_{t}$ affects the values of the dependent variable $v_{n, t, j}$. 


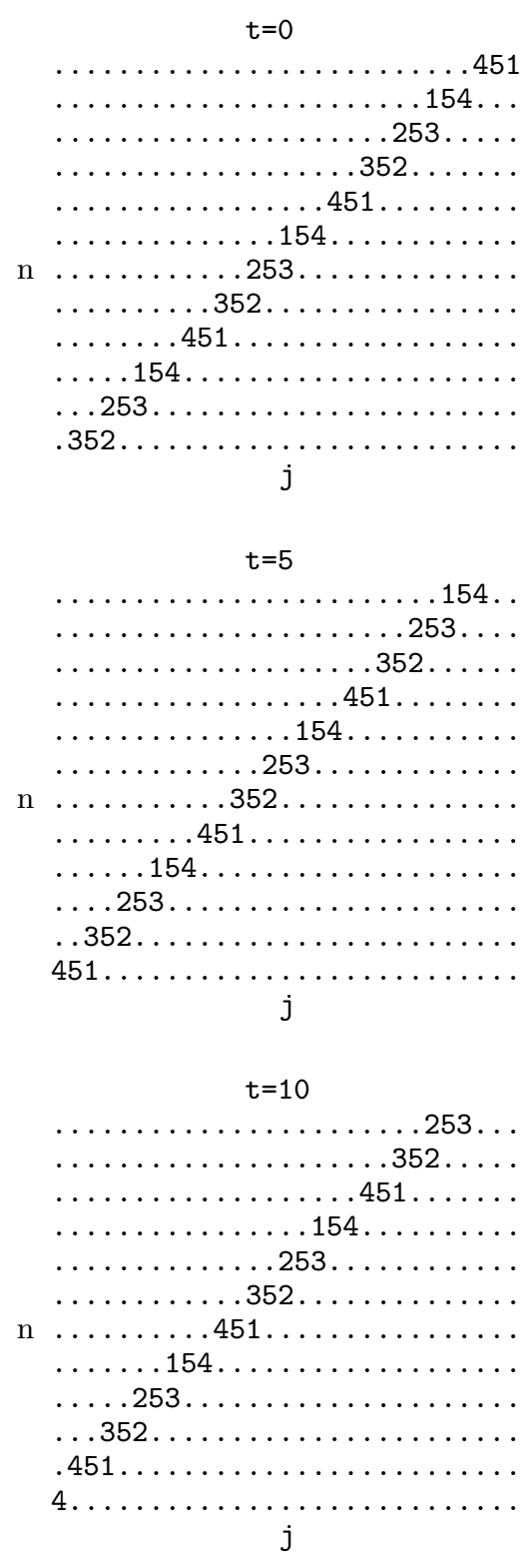

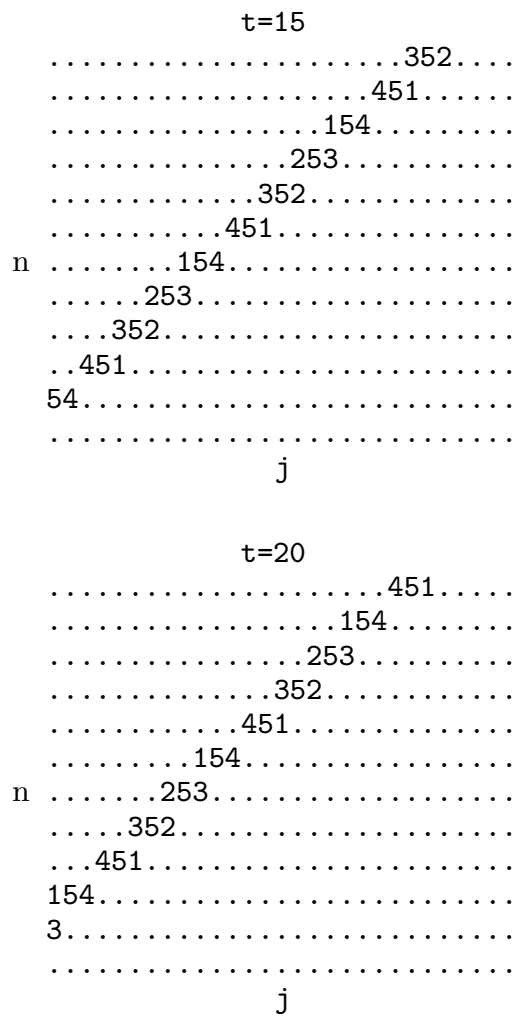

Figure 1: One-soliton solution for eq. (23) $\left(P_{1}=\right.$ $\left.-5, Q_{1}=4, \sigma_{j}=1, \theta_{t}=3\right)$. The value " 0 " is replaced by "." (period) for simplicity. 


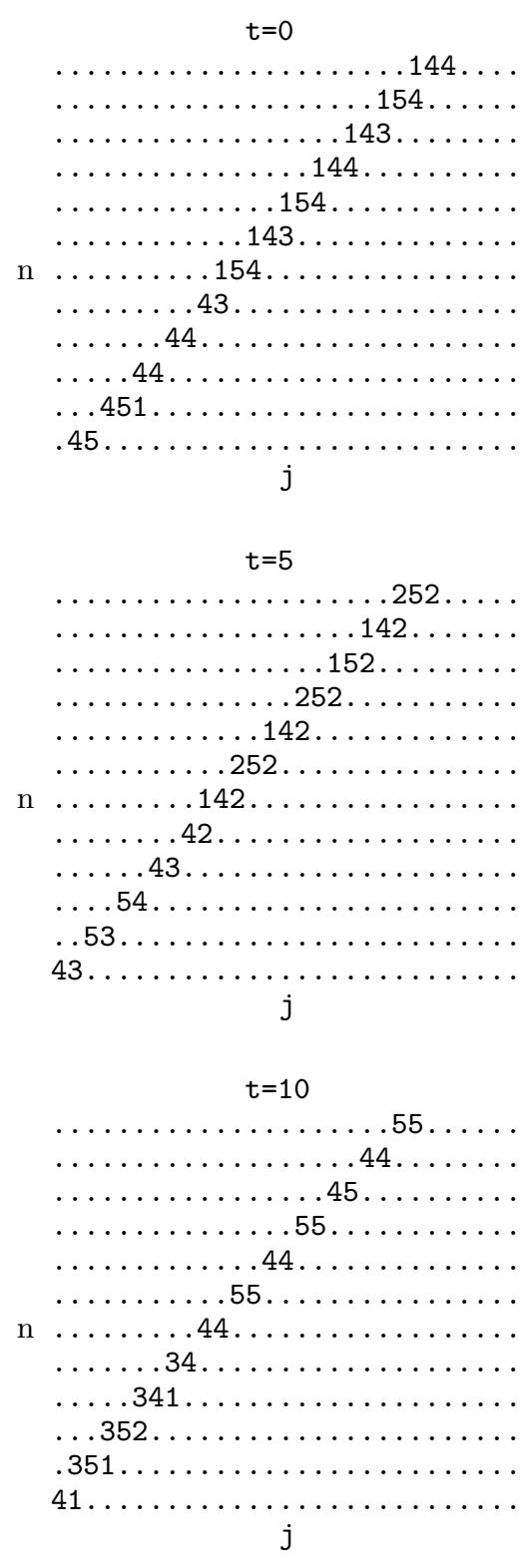

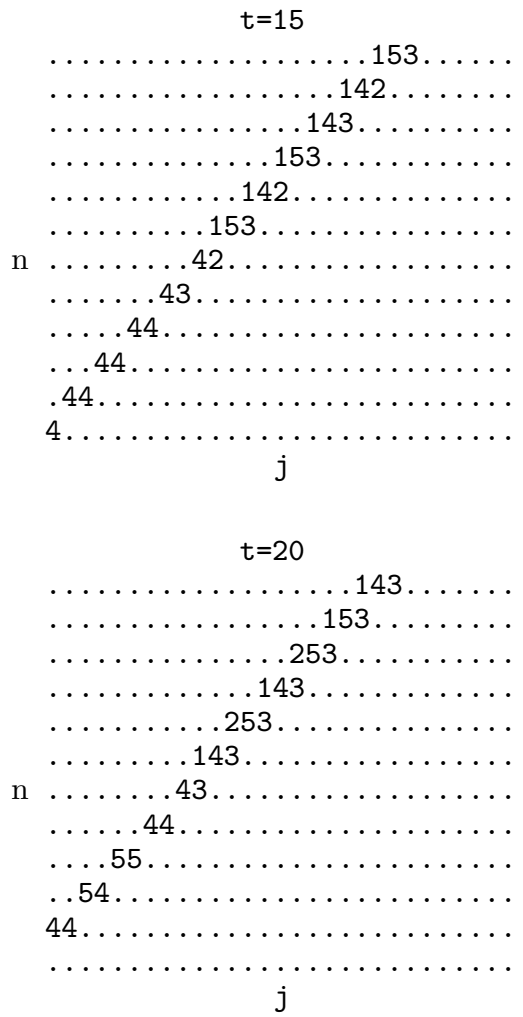

Figure 2: One-soliton solution for eq. (23) $\left(P_{1}=\right.$ $-5, Q_{1}=4, \sigma_{j}, \theta_{t}$ : randomly chosen). 

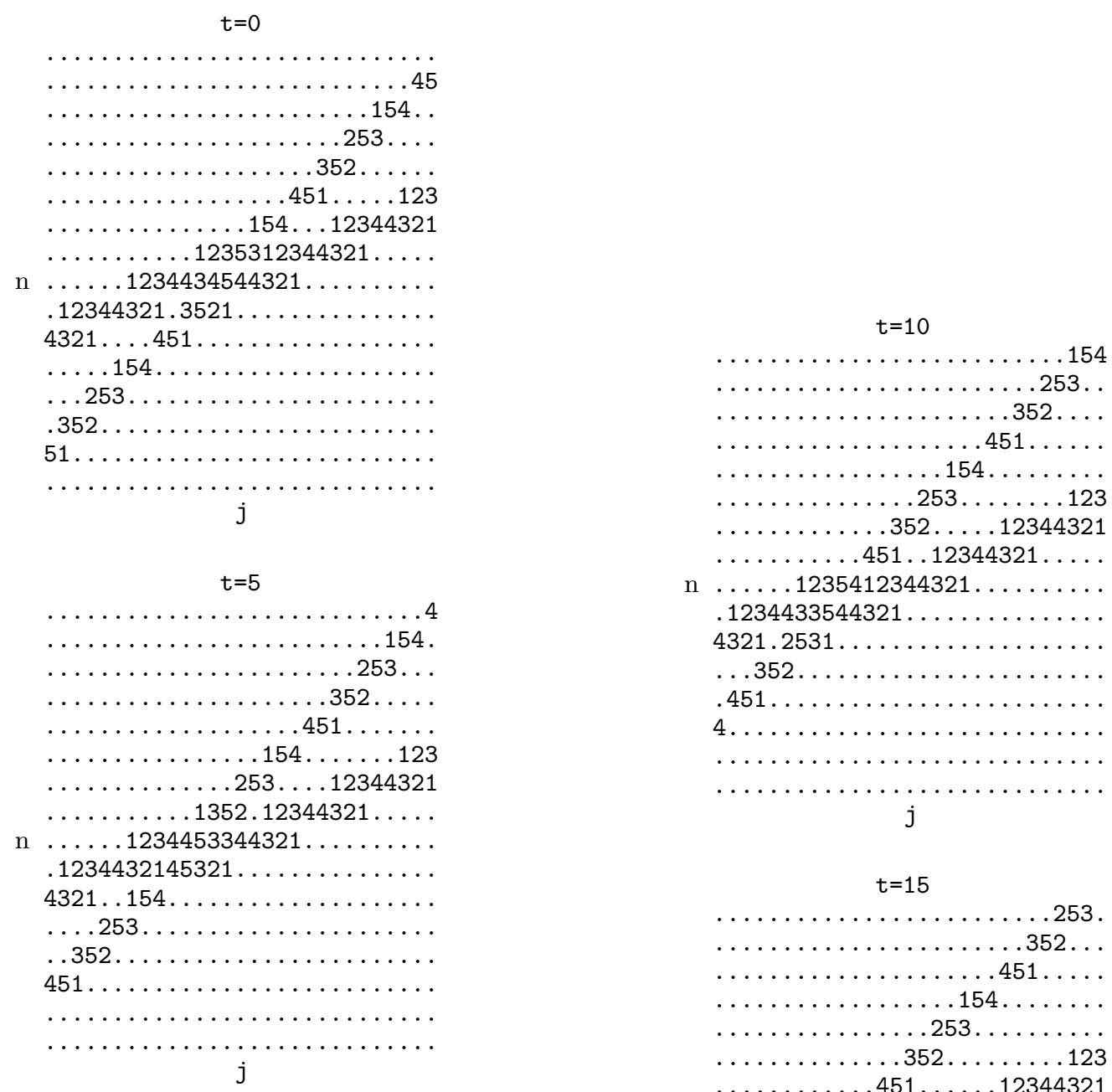

n . . . .1235412344321........

.1234433544321 .

4321.2531

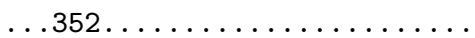

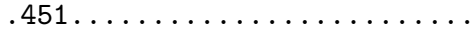

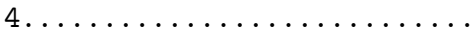

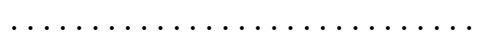

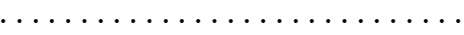

j

$\mathrm{t}=15$

....................

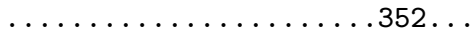

$\ldots \ldots \ldots \ldots \ldots \ldots 451 \ldots \ldots$

$\ldots \ldots \ldots \ldots \ldots \ldots$.

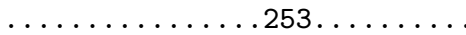

$\ldots \ldots \ldots \ldots 352 \ldots \ldots \ldots 123$

$\ldots \ldots \ldots \ldots 451 \ldots \ldots 12344321$

$\ldots \ldots \ldots 154 \ldots 12344321 \ldots \ldots$

n ....1253.12344321........

$.1234454344321 \ldots \ldots \ldots \ldots \ldots$

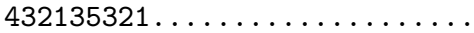

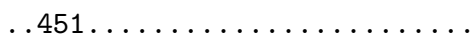

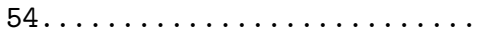

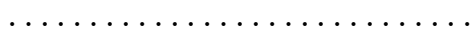

$\ldots \ldots \ldots \ldots \ldots \ldots \ldots \ldots$

j

Figure 3: Two-soliton solution for eq. 23) $\left(P_{1}=\right.$ $\left.-5, P_{2}=-2, Q_{1}=4, Q_{2}=3, \sigma_{j}=1, \theta_{t}=3\right)$ 


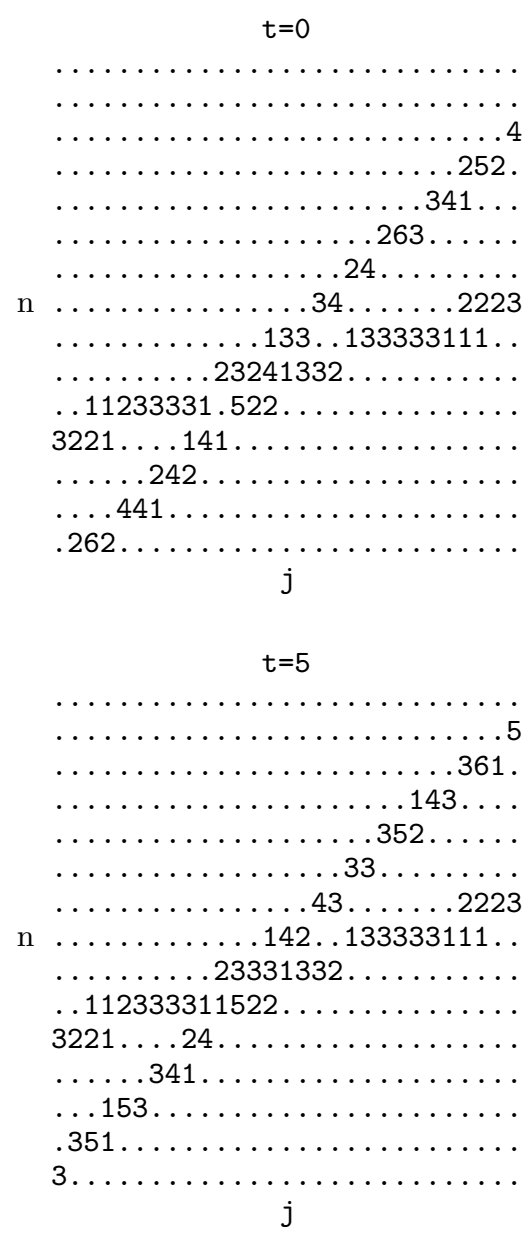

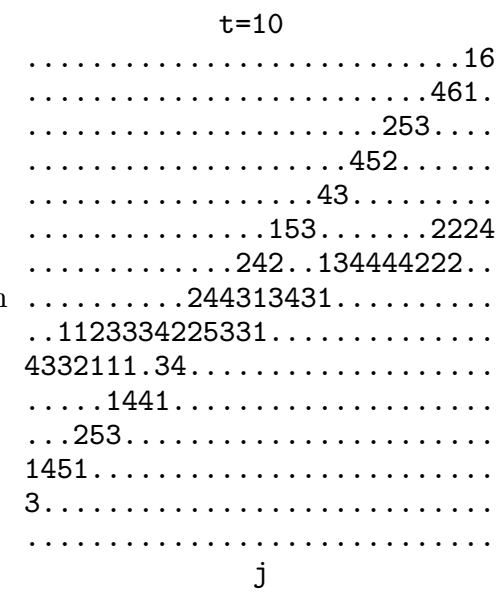

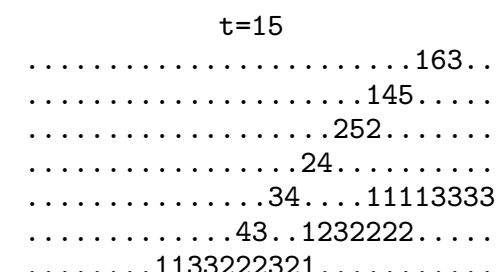

n $112233322.4311 \ldots \ldots \ldots \ldots$

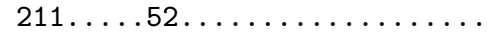

....361.

. .143

352 .

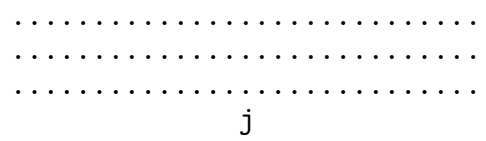

j

Figure 4: Two-soliton solution for eq. (23) $\left(P_{1}=-5, P_{2}=-2, Q_{1}=4, Q_{2}=3, \sigma_{j}, \theta_{t}:\right.$ randomly chosen) 


\section{Concluding Remarks}

We have presented a deautonomized 2D Toda lattice equation and its ultra-discrete analogue. We have also found soliton solutions for the latter case. The deautonomization resulting in eq. (23) causes arbitrariness in the values of the soliton solutions, a property which is not observed in the autonomous case. Deautonomization and ultra-discretization of discrete soliton equations are expected to find more applications in the field of computer science such as convergence acceleration, interpolation and sorting algorithms.

\section{Acknowledgements}

It is a pleasure to thank Professor Ryogo Hirota of Waseda University for fruitful discussions, especially on deautonomized soliton equations. One of the authors(R.W.) is a postdoctoral fellow of the Fund for Scientific Research(F. W. O.), Flanders (Belgium). He would also like to acknowledge the support of the F. W. O. through a mobility grant. The present work is partially supported by a Grant-in-Aid from the Japan Ministry of Education, Science and Culture.

\section{References}

[1] R. Hirota, J. Phys. Soc. Jpn. 67 (1997) 283.

[2] L. M. Milne-Thomson, The Calculus of Finite Differences, Chelsea, New York, 1933.

[3] D. Takahashi and J. Satsuma, J. Phys. Soc. Jpn. 59 (1990) 3514.

[4] D. Takahashi and J. Matsukidaira, Phys. Lett. A 209 (1995) 184.

[5] T. Tokihiro, D. Takahashi, J. Matsukidaira and J. Satsuma, Phys. Rev. Lett. 76 (1996) 3247.

[6] J. Matsukidaira, J. Satsuma, D. Takahashi, T. Tokihiro and M. Torii, Phys. Lett. A 225 (1997) 287.

[7] S. Moriwaki, A. Nagai, J. Satsuma, T. Tokihiro, M. Torii, D. Takahashi and J. Matsukidaira, to appear in London Math. Soc. Lecture Notes Series, Cambridge Univ. Press.

[8] R. Hirota, M. Ito and F. Kako, Prog. Theor. Phys. Suppl. 94 (1988) 42.

[9] R. Hirota, Direct Method in Soliton Theory (Iwanami, Tokyo, 1992) [in Japanese]. 